\title{
FACIAL EMOTION RECOGNITION AND THERORY OF MIND IN ELDERLY SUBJECTS LIVING IN INSTITUTIONS
}

\author{
A. Hakiri ${ }^{1}$, H. Ben Ammar ${ }^{1}$, R. Nefzi ${ }^{1}$, A. Aissa ${ }^{1}$, E. Khelifa ${ }^{1}$, Z. El Hechmi ${ }^{1}$. \\ ${ }^{1}$ Razi Psychiatric Hospital, psychiatric department "F", La Manouba, Tunisia.
}

Introduction: Social cognition refers to the cognitive abilities necessary for understanding and adapting to the social world. Recent data agrees on some reduction of social cognition with aging. Elderly institutionalized subjects experience a change in their social environment, and this change may have an impact on cognitive abilities and social adjustment.

Objectives: To assess two domains of social cognition which are : facial emotion recognition and the theory of mind in elderly subjects living in institutions.

Materials and Methods: A cross-sectional and descriptive study was conducted among 30 residents at the center of elderly subjects. The study included patients aged more than 65 years, not known as followed for having a dementia, a psychiatric disease, who does not have depressive or anxious symptoms at the moment of the study, and agreeing to participate.

Two dimensions of social cognition were evaluated.

The Facial Emotions Recognition (FER) adapted in tunisian dialect test is composed of 6 photos showing the 6 universally recognized emotions: happiness, sadness, fear, surprise, anger and disgust. theory of mind. It consists of 28 comics each showing a scenario
and where the subject must choose as quickly as possible the answer completing most logically the scenario answer among three possibilities.

the Mini Mental State Examination (MMSE) was used to evaluate global cognitive function.

\section{Results:}

The average age was 73.86 .3 [65-87].

$56.7 \%$ of subjects had a primary school level, all of them were retired, half of them have never been married and two thirds had no children. The average duration of stay in the center were 51 months Mean MMSE: 21.73 3.87 [14-29].

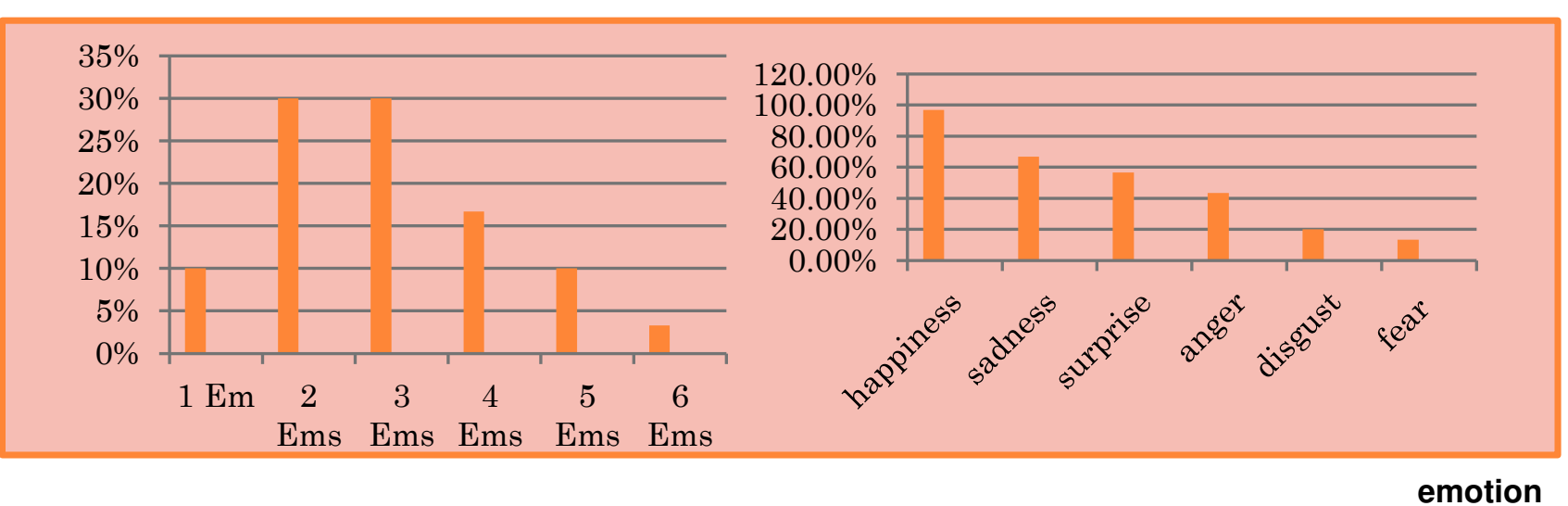

The average number of recognized emotions was $2.96 \pm 1.27$.

(Figures 1 and 2) Facial emotion recognition was significantly higher in subjects with the highest MMSE scores ( $p=0.017$ ).

The average number of correct responses on the IAT scale was $15.26 \pm 6.98$ with extremes of 0 and 27 for a total of 28 situations. Theory of mind was correlated with the FER $p=<0.001$ and the MMSE score $p<0.001$.

\section{Discussion:}

The rate of emotions recognition in our sample is lower than the rates reported in the literature, suggesting the role of institutionalization in the alteration of this dimension of social cognition in elderly subjects. Normal aging is accompanied by fronto-temporal deterioration, which explains the reduction in the capacity of RFE during aging.

Happiness was the most recognized emotion, fear and disgust were the least recognized, which joins previous studies. Indeed, during aging there is a stabilization or even an improvement of positive emotions such as happiness and a reduction in the recognition of negative emotions such as sadness, disgust and fear [1].

this was explained via neuroimaging, by reduced amygdala activation in negative facial expressions in older subjects.

In our study, we found a correlation between low MMSE scores and a reduced number of recognized emotions. This result is shared by previous studies. Indeed, it has been shown that facial recognition is impaired in moderate to severe cognitive deficits, and this deterioration increases with the evolution of the pathology [2,3]. we found an alteration of the theory of mind in subjects with low MMSE scores. Previous literature concluded that the theory of mind was severely affected by cognitive impairment especially in its cognitive component rather than the affective component.

This could be explained by the institutional life where social contacts are very limited to the staff of the establishment, other residents and some sporadic and irregular family visits

\section{Conclusion:}

The results suggest a progressive degradation of social cognition abilities during aging and this degradation is more important in case of cognitive decline. 